This paper deals with a distributed constraint optimization problem on networked multi-agent systems. First, we propose a distributed algorithm based on the Lagrangian method, where a new update law of the Lagrangian multiplier is designed. This update law enables each agent to estimate the value of the Lagrangian multiplier in a distributed manner. Next, we derive a necessary and sufficient condition that the optimization problem is solvable in a distributed manner over a graph. Finally, we apply the proposed method to power grid control via distributed pricing to maintain the supply-demand balance.

\section{Distributed Constraint Optimization on Networked Multi-Agent Systems}

\author{
${ }^{a}$ Kazunori Sakurama and Masashi Miura are with Graduate School of Engineering, \\ Tottori University, 4-101 Koyama-Minami, Tottori 680-8552 Japan. \\ sakurama@mech.tottori-u.ac.jp, miura@icee.tottori-u.ac.jp.
}

Keywords: distributed optimization, multi-agent system, Lagrangian method

\section{Introduction}

Recently, distributed optimization has been vigorously investigated to solve problems in large-scale systems. The most successful example is the Internet protocols $[1,2,3,4]$, where congestion control is implemented. Distributed control techniques are inevitable to reduce computation burdens in such an enormously large-scale system. In recent years, these techniques are becoming more and more important because the sizes of many public infrastructures are growing (e.g., power grids $[5,6,7,8]$ and traffic systems $[9,10,11])$ and the management of these systems is getting harder.

This paper deals with a distributed optimization problem in the terminology of networked multi-agent systems. Let $x_{i} \in \mathbb{R}^{d_{i}}$ be the variable updated by agent $i \in\{1,2, \ldots, n\}$. For $x=\left[\begin{array}{llll}x_{1}^{\top} & x_{2}^{\top} & \cdots & x_{n}^{\top}\end{array}\right]^{\top}$, let $F(x) \in \mathbb{R}$ and 
1 2

$G_{j}(x) \in \mathbb{R}, j=1,2, \ldots, m$ be the objective and constraint functions. Then, the constraint optimization problem is formulated as follows.

$$
\begin{cases}\text { minimize } & F(x) \\ \text { subject to } & G_{j}(x) \leq 0, j=1,2, \ldots, m\end{cases}
$$

Note that equality constraints can be easily handled by the choice of $G_{j}(x)$. Now, these agents can communicate with each other over a network $\mathcal{G}$ (which is described by a graph).

Then, each agent can update the value of $x_{i}$ by using only local information over $\mathcal{G}$. Let $x_{i}[k] \in \mathbb{R}^{d_{i}}$ be the updated value of $x_{i}$ at time $k$, and $\lambda_{j} \in \mathbb{R}$ be the Lagrangian multiplier (a kind of penalty) according to the constraint $G_{j}(x)$. Then, the distributed update law based on the Lagrangian method is of the form

$$
x_{i}[k+1]=f_{i}\left(x_{i}[k], x_{i_{1}}[k], x_{i_{2}}[k], \ldots, x_{i_{n_{i}}}[k], \lambda_{1}, \lambda_{2}, \ldots, \lambda_{m}\right),
$$

where $i_{1}, i_{2}, \ldots, i_{n_{i}}$ are the neighbors of agent $i$ over the network $\mathcal{G}$. (2) is distributed in the sense that the agent requires only its own, its neighbors' information and the Lagrangian multipliers.

Note that the Lagrangian multiplier $\lambda_{j}$ is common among the agents, hence it is global information. As long as the update law (2) requires the information on $\lambda_{j}$, a supervisor is inevitable to compute and broadcast the value of $\lambda_{j}$. In a large-scale system, a huge amount of information will be concentrated on the supervisor. Thus, the update law (2) is not completely distributed nor scalable. In order to make the update law (2) distributed, the value of $\lambda_{j}$ should be individually computed by each agent. However, generally, global information, the value of $G_{j}(x)$, is required to update $\lambda_{j}$. Is it possible to obtain the solution of (1) by distributed computations of not only $x_{i}$ but also $\lambda_{j}$ ?

In this paper, we propose a new distributed algorithm to solve the optimization problem $(1)$ over the network $\mathcal{G}$. Especially, we propose a distributed method to estimate the Lagrangian multiplier $\lambda_{j}$. By using this method, agents can individually obtain the estimated values of $\lambda_{j}$ through information exchange between the agents. Then, whether the solution of (1) is obtained or not depends on the structure of $\mathcal{G}$. So, we derive a necessary and sufficient condition of $\mathcal{G}$ with which the problem (1) is solvable in the distributed way. From this result, if an optimization problem is given, we 
can answer a strict class of networks with which the problem is distributedly solvable. Conversely, if a network is given, we can know what kind of optimization problem is solvable over this network.

As an application of the proposed method, we consider power grid control via pricing to maintain supply-demand balance. The Lagrangian multiplier $\lambda_{j}$ is corresponding to the electricity price. Our proposed method gives a basic rule to determine the price by negotiations between consumers, suppliers and distributors over a communication network.

\subsection{Contributions comparing to related researches}

Distributed optimization methods are summarized in [12]. For example, dual decomposition was developed in 1960s and has been modified by several researchers $[13,14,15,16]$. The main idea of the dual decomposition is to deal with separable functions with respect to agents, namely,

$$
\left\{\begin{array}{l}
\text { minimize } \quad \sum_{i=1}^{n} F_{i}\left(x_{i}\right) \\
\text { subject to } \quad \sum_{i=1}^{n} G_{i j}\left(x_{i}\right) \leq 0, j=1,2, \ldots, m
\end{array}\right.
$$

where $F_{i}\left(x_{i}\right)$ and $G_{i j}\left(x_{i}\right)$ depend on one agent's variable $x_{i}$. Then, the update law of each agent is given by

$$
x_{i}[k+1]=x_{i}[k]-\alpha\left(\frac{\partial F_{i}}{\partial x_{i}}\left(x_{i}[k]\right)+\sum_{j=1}^{m} \lambda_{j} \frac{\partial G_{i j}}{\partial x_{i}}\left(x_{i}[k]\right)\right),
$$

where $\alpha$ is a step gain. The update law (4) is of the form (2) because other agents' information is not required. However, this update law requires the global information $\lambda_{j}$. Moreover, the objective and constraint functions are restricted to separable forms as (3). The other primal-dual methods including alternating direction method of multipliers (ADMM), alternating direction augmented Lagrangian (ADAL) and so on $[17,18]$ have the same structure.

Recently, consensus-based distributed optimization has been investigated $[19,20,21,22,23]$. The consensus-based distributed optimization for the objective function $F(x)=\sum_{i=1}^{n} F_{i}(x)$ with agent-wise functions $F_{i}(x)$ is reduced to

$$
\left\{\begin{array}{l}
\text { minimize } \sum_{i=1}^{n} F_{i}\left(x_{i}\right) \\
\text { subject to } \quad x_{i}=x_{j}, i, j=1,2, \ldots, n
\end{array}\right.
$$



2 by by

$$
x_{i}[k+1]=x_{i}[k]-\alpha\left(\frac{\partial F_{i}}{\partial x_{i}}\left(x_{i}[k]\right)-\sum_{j \in \mathcal{N}_{i}}\left(x_{i}[k]-x_{j}[k]\right)\right),
$$

where $\mathcal{N}_{i}$ is the set of neighbors of agent $i$ over the network $\mathcal{G}$. The update law (6) is distributed and the solution is obtained only if $\mathcal{G}$ is connected.

Note that the objective function in (5) is separable, and other types of objective functions cannot be treated in this form.

Since these existing researches restrict optimization problems to the forms (3) and (5), the problems can be solved by the distributed update laws (4) and (6) (although (4) is not completely distributed because of $\lambda_{j}$ ). The question here is that if we do not restrict optimization problems to these forms, what kind of problems can be solved in a distributed manner over the network $\mathcal{G}$ ? Are there more problems solvable in a distributed manner? To answer these questions, we give a complete characterization of the class of optimization problems solvable in a distributed manner over $\mathcal{G}$. The derived class indicates the limit of the graph $\mathcal{G}$ 's ability to solve distributed optimization problems. As far as the authors' knowledge, this is the first paper which specifies the graph's ability in this way. As a result, this paper reveals that we can solve a wider class of optimization problems than (3) and (5) in a distributed manner. See Example 1 and Section 4 for such examples.

\subsection{Notations}

The following notations are used in this paper. Let $\mathbb{R}, \mathbb{R}_{+}$and $\mathbb{Z}_{+}$be the sets of the real numbers, nonnegative real numbers and nonnegative integers. The set of $n$-dimensional (non-negative) real numbers is represented as $\mathbb{R}^{n}\left(\mathbb{R}_{+}^{n}\right)$. The cardinal number of a set is described as $|\cdot|$. For a set $\mathcal{S} \subset\{1,2, \ldots, n\}$ with the cardinal number $|\mathcal{S}|=m$, there exists a strictly increasing sequence $s_{1}, s_{2}, \ldots, s_{m} \leq n$ of natural numbers such that $\mathcal{S}=$ $\left\{s_{1}, s_{2}, \ldots, s_{m}\right\}$. For $\mathcal{S}$ and $x_{i} \in \mathbb{R}^{d_{i}}, i=1,2, \cdots, n$, we use the notation $x_{\mathcal{S}}=\left[\begin{array}{llll}x_{s_{1}}^{\top} & x_{s_{2}}^{\top} & \cdots & x_{s_{m}}^{\top}\end{array}\right]^{\top}$. Let the function $[\cdot]^{+}: \mathbb{R}^{n} \rightarrow \mathbb{R}_{+}^{n}$ be the operation of replacing negative components of a vector with zero such that

$$
\left([x]^{+}\right)_{i}=\left\{\begin{array}{ll}
x_{i} & \text { if } x_{i} \geq 0 \\
0 & \text { if } x_{i}<0
\end{array},\right.
$$

where $\left([x]^{+}\right)_{i} \in \mathbb{R}_{+}$is the $i$-th component of the vector $[x]^{+} \in \mathbb{R}_{+}^{n}$. 


\section{Problem setting}

\subsection{Lagrangian method}

Consider the optimization problem (1). We assume that functions $F(x)$ and $G_{j}(x) \in \mathbb{R}, j=1,2, \ldots, m$ for $x \in \mathbb{R}^{N}$ are continuously differentiable.

The Lagrangian method is introduced to solve (1) [24]. Let

$$
L(x, \lambda)=F(x)+\lambda^{\top} G(x)
$$

be the Lagrangian function with the Lagrangian multiplier $\lambda \in \mathbb{R}_{+}^{m}$ and the constraint function $G(x)=\left[\begin{array}{llll}G_{1}(x) & G_{2}(x) & \cdots & G_{m}(x)\end{array}\right]^{\top}$. Let

$$
\Phi(\lambda)=\min _{x \in \mathbb{R}^{N}} L(x, \lambda)
$$

be the Lagrangian dual function. Then,

$$
\left\{\begin{array}{l}
\text { maximize } \Phi(\lambda) \\
\text { subject to } \lambda \geq 0
\end{array}\right.
$$

is called the dual problem of the primal problem (1).

If the solution $\lambda$ of (9) is achieved, the solution $x$ of (1) is obtained from (8) under the assumption of the strong duality. To obtain the solution based on the dual problem, we update $x$ so as to decrease $L(x, \lambda)$ from (8), then update $\lambda$ so as to increase $L(x, \lambda)$ according to (9), and repeat these updates alternatively. An algorithm based on this method is given as follows.

Algorithm A:

(A.1) Give the initial vectors $x^{[0]} \in \mathbb{R}^{N}, \lambda^{[0]} \in \mathbb{R}_{+}^{m}$ and constants $\alpha, \beta>0$. Set the step $s=0$.

(A.2) Set $x[0]=x^{[s]} \in \mathbb{R}^{N}$ and execute the recursive computation

$$
x[k+1]=x[k]-\alpha\left(\frac{\partial F}{\partial x}(x[k])+\left(\lambda^{[s]}\right)^{\top} \frac{\partial G}{\partial x}(x[k])\right)
$$

for $k \in \mathbb{Z}_{+}$. Then, update $x^{[s]}$ to $x^{[s+1]}=\lim _{k \rightarrow \infty} x[k]$.

(A.3) Update $\lambda^{[s]} \in \mathbb{R}_{+}^{m}$ to

$$
\lambda^{[s+1]}=\left[\lambda^{[s]}+\beta G\left(x^{[s+1]}\right)\right]^{+} .
$$

(A.4) Add 1 to the step $s$ and return to (A.2). 
The update variable $x^{[s]}$ converges to the solution of (1) under two assumptions. The first assumption is a certain relaxed condition to guarantee the strong duality. Then, there is no gap between the solutions of the primal and dual problems. The second assumption is the twise continuous differentiability and the convexity of the functions $F(x)$ and $G_{j}(x), j=1,2, \ldots, m$. This is to achieve the global minimum by using the gradient-based methods. We assume these conditions hereafter.

Remark 1. In the iteration of (A.2) of the algorithm, the limit $\lim _{k \rightarrow \infty} x[k]$ exists under the assumptions of the functions $F(x)$ and $G(x)$. On the other hand, in practice, the iteration has to be truncated, so there is numerical error to the $\operatorname{limit}_{k \rightarrow \infty} x[k]$. In this case, we obtain the update variable $x^{[s+1]}=\lim _{k \rightarrow \infty} x[k]+\varepsilon^{[s+1]}$ with the error $\varepsilon^{[s+1]}$. Then, the optimization error remains in proportion to the absolute value of $\varepsilon^{[s+1]}$ and its square [25]. Therefore, the optimization error can be arbitrarily small as the number of the iteration increases.

\subsection{Distributed update}

Consider a multi-agent system with $n$ agents over the network represented by a graph $\mathcal{G}=(\mathcal{V}, \mathcal{E})$, where the node set $\mathcal{V}=\{1,2, \ldots, n\}$ and the edge set $\mathcal{E}$ represent the group of agents and their network connections, respectively. Assume that $\mathcal{G}$ is simple and undirected, and the edge between $i, j \in \mathcal{V}$ is represented as $\{i, j\} \in \mathcal{E}$. Let $x_{i} \in \mathbb{R}^{d_{i}}$ be the variable updated by agent $i$. Our goal is to solve the constraint optimization problem (1) in a distributed manner over the graph $\mathcal{G}$, where $N=\sum_{i}^{n} d_{i}$.

Now, we try to employ Algorithm A for this purpose. The problem here is that the Lagrangian multiplier $\lambda$ is global information. Therefore, the value $\lambda^{[s]} \in \mathbb{R}_{+}^{m}$ in Algorithm A should be updated and broadcast by a supervisor. Our stance in this paper is to adopt a completely distributed manner without supervisors. To attain this, we let each agent estimate the value of $\lambda^{[s]} \in \mathbb{R}_{+}^{m}$ individually. The estimate value of agent $i$ is described as $\hat{\lambda}_{i}^{[s]} \in \mathbb{R}_{+}^{m}$. Now, agent $i$ has to update two variables $x_{i}^{[s]}$ and $\hat{\lambda}_{i}^{[s]}$ in a distributed manner over $\mathcal{G}$.

Let $x_{i}[k] \in \mathbb{R}^{d_{i}}$ and $\hat{\lambda}_{i}[l] \in \mathbb{R}^{m}$ be the temporal update values for $x_{i}^{[s]}$ and $\hat{\lambda}_{i}^{[s]}$. Let $\mathcal{N}_{i}$ be the set of neighbors of agent $i$ over $\mathcal{G}$ as

$$
\mathcal{N}_{i}=\{j \in \mathcal{V} \mid\{i, j\} \in \mathcal{E}\}
$$


Consider some functions $f_{i}: \mathbb{R}^{d_{i}} \times \mathbb{R}^{D_{i}} \times \mathbb{R}^{d_{i}} \times \mathbb{R}^{D_{i}} \times \mathbb{R}^{m} \times \mathbb{R}^{m\left|\mathcal{N}_{i}\right|} \rightarrow \mathbb{R}^{d_{i}}$, $g_{i}: \mathbb{R}^{d_{i}} \times \mathbb{R}^{D_{i}} \times \mathbb{R}^{m} \times \mathbb{R}^{m\left|\mathcal{N}_{i}\right|} \rightarrow \mathbb{R}^{m}$, and $h_{i}: \mathbb{R}^{m} \times \mathbb{R}^{m\left|\mathcal{N}_{i}\right|} \times \mathbb{R}^{d_{i}} \times \mathbb{R}^{D_{i}} \times \mathbb{R}^{m} \times$ $\mathbb{R}^{m\left|\mathcal{N}_{i}\right|} \rightarrow \mathbb{R}^{m}$ where $D_{i}=\sum_{j \in \mathcal{N}_{i}} d_{j}$. Then, an algorithm to update $x_{i}^{[s]}$ and $\hat{\lambda}_{i}^{[s]}$ in a distributed manner is described as follows.

Algorithm B:

(B.1) Each agent gives initial vectors $x_{i}^{[0]} \in \mathbb{R}^{d_{i}}$ and $\hat{\lambda}_{i}^{[0]} \in \mathbb{R}_{+}^{m}$. Set the step $s=0$.

(B.2) Each agent sets $x_{i}[0]=x_{i}^{[s]}$, executes

$$
x_{i}[k+1]=f_{i}\left(x_{i}[k], x_{\mathcal{N}_{i}}[k], x_{i}^{[s]}, x_{\mathcal{N}_{i}}^{[s]}, \hat{\lambda}_{i}^{[s]}, \hat{\lambda}_{\mathcal{N}_{i}}^{[s]}\right)
$$

for $k \in \mathbb{Z}_{+}$, and updates $x_{i}^{[s]}$ to $x_{i}^{[s+1]}=\lim _{k \rightarrow \infty} x_{i}[k]$.

(B.3) Each agent sets

$$
\hat{\lambda}_{i}[0]=g_{i}\left(x_{i}^{[s+1]}, x_{\mathcal{N}_{i}}^{[s+1]}, \hat{\lambda}_{i}^{[s]}, \hat{\lambda}_{\mathcal{N}_{i}}^{[s]}\right)
$$

and executes

$$
\hat{\lambda}_{i}[l+1]=h_{i}\left(\hat{\lambda}_{i}[l], \hat{\lambda}_{\mathcal{N}_{i}}[l], x_{i}^{[s+1]}, x_{\mathcal{N}_{i}}^{[s+1]}, \hat{\lambda}_{i}^{[s]}, \hat{\lambda}_{\mathcal{N}_{i}}^{[s]}\right)
$$

for $l \in \mathbb{Z}_{+}$, and updates $\hat{\lambda}_{i}^{[s]}$ to $\hat{\lambda}_{i}^{[s+1]}=\left[\lim _{l \rightarrow \infty} \hat{\lambda}_{i}[l]\right]^{+}$.

(B.4) Add 1 to the step $s$ and return to (B.2).

In this paper, we say an algorithm is distributed over the graph $\mathcal{G}$ if the computations of each agent in the algorithm is given by a function depending on its own information and the neighbors' one. In Algorithm $\mathrm{B}$, agent $i$ uses only its own variable $x_{i}[k], \hat{\lambda}_{i}[l], x_{i}^{[s]}, x_{i}^{[s+1]}, \hat{\lambda}_{i}^{[s]}$ and the neighbors' variables $x_{\mathcal{N}_{i}}[k], \hat{\lambda}_{\mathcal{N}_{i}}[l], x_{\mathcal{N}_{i}}^{[s]}, x_{\mathcal{N}_{i}}^{[s+1]}, \hat{\lambda}_{\mathcal{N}_{i}}^{[s]}$. In this sense, this algorithm is distributed.

Now, we design functions $f_{i}, g_{i}$ and $h_{i}$ such that Algorithm B works as Algorithm A. Then, the solution of the constraint optimization problem (1) is obtained in a distributed manner. Do there really exist such functions $f_{i}$, $g_{i}$ and $h_{i}$ ? Actually, the feasibility depends on the structure of the graph $\mathcal{G}$ because it determines the neighbors $\mathcal{N}_{i}$ in (13), (14) and (15). Now, the main problem is formulated as follows.

Problem 1. For continuously differentiable functions $F: \mathbb{R}^{N} \rightarrow \mathbb{R}$ and $G: \mathbb{R}^{N} \rightarrow \mathbb{R}^{m}$ and a graph $\mathcal{G}$, derive a necessary and sufficient condition such that there exist continuous functions $f_{i}, g_{i}$ and $h_{i}$ and positive constants $\alpha, \beta$ satisfying the following conditions. 
(i) (13) is equivalent to

$$
x_{i}[k+1]=x_{i}[k]-\alpha\left(\frac{\partial F}{\partial x_{i}}(x[k])+\left(\hat{\lambda}_{i}^{[s]}\right)^{\top} \frac{\partial G}{\partial x_{i}}(x[k])\right) .
$$

(ii) (14) and (15) generate $\hat{\lambda}_{i}^{[s+1]}=\left[\lim _{l \rightarrow \infty} \hat{\lambda}_{i}[l]\right]^{+}$such that

$$
\begin{aligned}
& \lambda_{i}^{[s+1]}=\left[\hat{\lambda}_{i}^{[s]}+\beta G\left(x^{[s+1]}\right)\right]^{+} \\
& \hat{\lambda}_{i}^{[s+1]}=\hat{\lambda}_{j}^{[s+1]}, \forall i, j \in \mathcal{V} .
\end{aligned}
$$

Moreover, design these functions $f_{i}, g_{i}$ and $h_{i}$.

The big difference between Algorithms A and B is that the latter is based on the agent-wise variables $\hat{\lambda}_{i}^{[s]}$ as a Lagrangian multiplier. However, if Problem 1 is solved, then $\hat{\lambda}_{i}^{[s]}$ are updated into the same value among all agents as (18), and each agent can use it as a Lagrangian multiplier because of (17). Then, Algorithm B works as Algorithm A and we can obtain the solution of the constraint optimization problem (1) in a distributed manner.

Remark 2. It seems that the update variable $\hat{\lambda}_{i}^{[s+1]}$ is possibly obtained by the direct computation of (17). However, to do so, all information on $x^{[s+1]}$ related to $G\left(x^{[s+1]}\right)$ is necessary. Therefore, it is not distributed anymore. Instead, we update $\hat{\lambda}_{i}^{[s]}$ in a distributed manner through the repetitive calculation (14) and (15). We expect that $\hat{\lambda}_{i}^{[s]}$ is updated into the same value satisfying (17) as a result.

Remark 3. The concept of the distributedness here is different from the other primal-dual methods as ADMM and ADAL [17, 18]. These existing methods are said to be distributed if the variable $x_{i}$ of each agent can be updated by using its own information and the information on the Lagrange multiplier $\lambda$. Note that $\lambda$ is updated by a supervisor and that the information on neighbors is not used. On the other hand, in our paper, we assume that there is no supervisor who updates $\lambda$, but instead the agents individually estimate the value of $\lambda$ through communication between neighbors. 


\section{Main result}

First, consider condition (i) in Problem 1. (13) and (16) are equivalent

${ }_{3}$ if and only if there exists a continuous function $\hat{f}_{i}: \mathbb{R}^{d_{i}} \times \mathbb{R}^{m} \times \mathbb{R}^{D_{i}} \rightarrow \mathbb{R}^{d_{i}}$ 4 such that

$$
\frac{\partial F}{\partial x_{i}}(x)+\hat{\lambda}_{i}^{\top} \frac{\partial G}{\partial x_{i}}(x) \equiv \hat{f}_{i}\left(x_{i}, \hat{\lambda}_{i}, x_{\mathcal{N}_{i}}\right) .
$$

${ }_{5}$ Let $\mathcal{S}_{\text {clq }}(\mathcal{G})$ be the set of all cliques on the graph $\mathcal{G}$. A clique on $\mathcal{G}$ is a node 6 subset $\mathcal{I} \subset \mathcal{V}$ such that the subgraph induced by $\mathcal{I}$ is complete. Therefore, $7 \mathcal{S}_{\mathrm{clq}}(\mathcal{G})$ is described as follows.

$$
\mathcal{S}_{\mathrm{clq}}(\mathcal{G})=\{\mathcal{I} \subset \mathcal{V} \mid\{i, j\} \in \mathcal{E} \quad \forall i, j \in \mathcal{V}, i \neq j\}
$$

8 Then, the necessary and sufficient condition for (19) is derived from the 9 authors' paper [26].

10 Lemma 1. For continuously differentiable functions $F: \mathbb{R}^{N} \rightarrow \mathbb{R}$ and $G$ : $\mathbb{R}^{N} \rightarrow \mathbb{R}^{m}$, a vector $x=\left[\begin{array}{llll}x_{1}^{\top} & x_{2}^{\top} & \cdots & x_{n}^{\top}\end{array}\right]^{\top} \in \mathbb{R}^{N}$ with $x_{i} \in \mathbb{R}^{d_{i}}$ and a graph $\mathcal{G}$, 12 there exist a continuous function $\hat{f}_{i}: \mathbb{R}^{d_{i}} \times \mathbb{R}^{m} \times \mathbb{R}^{D_{i}} \rightarrow \mathbb{R}^{d_{i}}$ for any $i \in \mathcal{V}$ 13 satisfying (19) if and only if there exist continuously differentiable functions ${ }_{14} \quad \bar{F}^{\mathcal{I}}: \mathbb{R}^{\sum_{j \in \mathcal{I}} d_{j}} \rightarrow \mathbb{R}$ and $\bar{G}^{\mathcal{I}}: \mathbb{R}^{\sum_{j \in \mathcal{I}} d_{j}} \rightarrow \mathbb{R}^{m}$ for $\mathcal{I} \in \mathcal{S}_{\text {clq }}(\mathcal{G})$ such that

$$
\begin{aligned}
F(x) & =\sum_{\mathcal{I} \in \mathcal{S}_{\mathrm{clq}}(\mathcal{G})} \bar{F}^{\mathcal{I}}\left(x_{\mathcal{I}}\right) \\
G(x) & =\sum_{\mathcal{I} \in \mathcal{S}_{\mathrm{clq}}(\mathcal{G})} \bar{G}^{\mathcal{I}}\left(x_{\mathcal{I}}\right) .
\end{aligned}
$$

15

16 Because $F(x)$ and $G(x)$ have to be of the forms (21) and (22), the update 17 law (16) is reduced to

$$
x_{i}[k+1]=x_{i}[k]-\alpha \sum_{\mathcal{I} \in \mathcal{S}_{\mathrm{clq}, i}(\mathcal{G})}\left(\frac{\partial \bar{F}^{\mathcal{I}}}{\partial x_{i}}\left(x_{\mathcal{I}}[k]\right)+\left(\hat{\lambda}_{i}^{[s]}\right)^{\top} \frac{\partial \bar{G}^{\mathcal{I}}}{\partial x_{i}}\left(x_{\mathcal{I}}[k]\right)\right),
$$

${ }_{18}$ where $\mathcal{S}_{\text {clq }, i}(\mathcal{G})$ is the set of the cliques including $i$ as follows.

$$
\mathcal{S}_{\mathrm{clq}, i}(\mathcal{G})=\left\{\mathcal{I} \in \mathcal{S}_{\mathrm{clq}}(\mathcal{G}) \mid i \in \mathcal{I}\right\}
$$


1 Note that (23) is of the distributed form (13) because $j \in \mathcal{N}_{i}$ holds for any $j \in \mathcal{I} \in \mathcal{S}_{\mathrm{clq}, i}(\mathcal{G})$.

Next, consider condition (ii) in Problem 1. Because $G(x)$ is of the form (22), (17) is reduced to

$$
\hat{\lambda}_{i}^{[s+1]}=\left[\hat{\lambda}_{i}^{[s]}+\beta \sum_{\mathcal{I} \in \mathcal{S}_{\mathrm{clq}}(\mathcal{G})} \bar{G}^{\mathcal{I}}\left(x_{\mathcal{I}}^{[s+1]}\right)\right]^{+} .
$$

It is impossible to directly compute the right-hand side of (25) in a distributed manner because agent $i$ needs the information on the variables $x_{\mathcal{I}}^{[s+1]}$ for all the cliques $\mathcal{I} \in \mathcal{S}_{\text {clq }}(\mathcal{G})$. The information that agent $i$ can obtain is at most the variables $x_{\mathcal{I}}^{[s+1]}$ for the cliques $\mathcal{I} \in \mathcal{S}_{\mathrm{clq}, i}(\mathcal{G})$ which he belongs to. From this viewpoint, we propose the following update law of $\hat{\lambda}_{i}^{[s]}$ :

$$
\begin{aligned}
\hat{\lambda}_{i}[0] & =\hat{\lambda}_{i}^{[s]}+\kappa \sum_{\mathcal{I} \in \mathcal{S}_{\mathrm{clq}, i}(\mathcal{G})} \gamma^{\mathcal{I}} \bar{G}^{\mathcal{I}}\left(x_{\mathcal{I}}^{[s+1]}\right) \\
\hat{\lambda}_{i}[l+1] & =\hat{\lambda}_{i}[l]-\eta \sum_{j \in \mathcal{N}_{i}}\left(\hat{\lambda}_{i}[l]-\hat{\lambda}_{j}[l]\right)
\end{aligned}
$$

for some positive constants $\kappa, \gamma^{\mathcal{I}}$ and $\eta$. Then, (26) and (27) are of the distributed forms (14) and (15). Note that (27) is a consensus controller, hence all $\hat{\lambda}_{i}[l]$ converge to the same value if $\mathcal{G}$ is connected and $\eta<1 /\left|\mathcal{N}_{i}\right|$ holds for all $i \in \mathcal{V}$ [27]. Thus, (18) is achieved. Moreover, (17) is obtained as proved in the following theorem. The main result of this paper is derived as follows.

Theorem 1. Consider continuously differentiable functions $F: \mathbb{R}^{N} \rightarrow \mathbb{R}$, $G: \mathbb{R}^{N} \rightarrow \mathbb{R}^{m}$ and a graph $\mathcal{G}$. There exist functions $f_{i}, g_{i}, h_{i}$ and positive constants $\alpha, \beta$ such that (i) (13) and (16) are equivalent, (ii) (14) and (15) generate $\hat{\lambda}_{i}^{[s+1]}=\left[\lim _{l \rightarrow \infty} \hat{\lambda}_{i}[l]\right]^{+}$satisfying (17) and (18) if and only if $\mathcal{G}$ is connected and there exist functions $\bar{F}^{\mathcal{I}}\left(x_{\mathcal{I}}\right)$ and $\bar{G}^{\mathcal{I}}\left(x_{\mathcal{I}}\right)$ for $\mathcal{I} \in \mathcal{S}_{\text {clq }}(\mathcal{G})$ such that $F(x)$ and $G(x)$ are of the forms (21) and (22). Under these conditions, such $f_{i}, g_{i}, h_{i}$ are given by the right-hand sides of (23), (26) and (27) for $\gamma^{\mathcal{I}}=1 /|\mathcal{I}|, \kappa>0$ and a positive constant $\eta<1 /\left|\mathcal{N}_{i}\right|$ for all $i \in \mathcal{V}$.

Proof. To show the sufficiency, assume that $\mathcal{G}$ is connected and that $F(x)$ and $G(x)$ are of the forms (21) and (22). Then, (i) is satisfied from Lemma 1. 
1 As for (ii), (18) is achieved because (27) is a consensus controller. Moreover, $2 \hat{\lambda}_{i}[l]$ converges to the average of the initial states $\hat{\lambda}_{j}[l]$ for all agents $j \in \mathcal{V}$ 3 under the assumption that $\eta<1 /\left|\mathcal{N}_{i}\right|[27]$. Thus, from (26) and $\gamma^{\mathcal{I}}=1 /|\mathcal{I}|$, 4 the following is derived.

$$
\begin{aligned}
\lim _{l \rightarrow \infty} \hat{\lambda}_{i}[l] & =\frac{1}{n} \sum_{j=1}^{n} \hat{\lambda}_{j}[0] \\
& =\frac{1}{n} \sum_{j=1}^{n}\left(\hat{\lambda}_{j}^{[s]}+\kappa \sum_{\mathcal{I} \in \mathcal{S}_{\mathrm{clq}, j}(\mathcal{G})} \frac{1}{|\mathcal{I}|} \bar{G}^{\mathcal{I}}\left(x_{\mathcal{I}}^{[s+1]}\right)\right) \\
& =\frac{1}{n} \sum_{j=1}^{n} \hat{\lambda}_{j}^{[s]}+\frac{\kappa}{n} \sum_{\mathcal{I} \in \mathcal{S}_{\mathrm{clq}}(\mathcal{G})} \sum_{j \in \mathcal{I}} \frac{1}{|\mathcal{I}|} \bar{G}^{\mathcal{I}}\left(x_{\mathcal{I}}^{[s+1]}\right) \\
& =\bar{\lambda}^{[s]}+\beta \sum_{\mathcal{I} \in \mathcal{S}_{\mathrm{clq}}(\mathcal{G})} \bar{G}^{\mathcal{I}}\left(x_{\mathcal{I}}^{[s+1]}\right),
\end{aligned}
$$

5 where

$$
\bar{\lambda}^{[s]}=\frac{1}{n} \sum_{j=1}^{n} \hat{\lambda}_{j}^{[s]}, \beta=\frac{\kappa}{n} .
$$

6 From the update of the previous step, $\hat{\lambda}_{i}^{[s]}=\hat{\lambda}_{j}^{[s]}$ hold for all $j \in \mathcal{V}$, hence $7 \bar{\lambda}^{[s]}=\hat{\lambda}_{i}^{[s]}$ holds. Therefore, from (22) and (28), we obtain (17) for $\hat{\lambda}_{i}^{[s+1]}=$ $8\left[\lim _{l \rightarrow \infty} \hat{\lambda}_{i}[l]\right]^{+}$.

9 The necessity is shown. If $\mathcal{G}$ is not connected, consensus is not achieved 10 [27]. Thus, (18) is not realized. If either $F(x)$ or $G(x)$ is not of the form (21) or (22), then (16) is not of the form (13) from Lemma 1. The proof is completed.

Example 1. Consider the functions

$$
\begin{aligned}
& F(x)=\left(x_{1}+x_{2}\right)^{2}+\left(x_{2}+x_{3}\right)^{2}+\left(x_{3}+x_{4}+x_{5}\right)^{4} \\
& G(x)=\left[\begin{array}{l}
x_{1}+x_{3} \\
x_{3}+x_{5}
\end{array}\right] .
\end{aligned}
$$

14 Then, $F(x)$ is of the form (21) if and only if the $\mathcal{G}$ contains the cliques $15\{1,2\},\{2,3\},\{3,4,5\} . G(x)$ is of the form (22) if and only if the $\mathcal{G}$ contains 16 the cliques $\{1,3\}$ and $\{3,5\}$. Such a graph is depicted in Fig. 1. Therefore, 
in order to update $x_{i}^{[s]}$ in a distributed manner, the graph $\mathcal{G}$ has to contain at least the edges in Fig. 1. Moreover, such $\mathcal{G}$ is connected. Then, Theorem 1 guarantees that the optimization problem is solvable in the distributed way.

Theorem 1 gives the strict class of graphs $\mathcal{G}$ with which the optimization problem (1) is solvable in the distributed way. Thus, if $\mathcal{G}$ is not in this class, we can say that $\mathcal{G}$ has to be reconstructed. Conversely, if a connected graph $\mathcal{G}$ is given in advance, Theorem 1 gives the strict classes of functions $F(x)$ and $G(x)$ solvable in the distributed way. These classes are given by (21) and (22), which indicate the limit of the graph $\mathcal{G}$ 's ability to solve the optimization problem. Note that the problem (1) with (21) and (22) contains a wider class of optimization problems than the conventional ones as (3) and (5). Actually, Example 1 is not of the conventional forms.

\section{Application to control of power system}

Consider the power grid model depicted in Fig. 2. The communication network between the agents is given by Fig. 3. There are three types of agents in this grid: consumers, suppliers and distributors. Agents $i=1,2, \cdots, 17$ have the variables $x_{i} \in \mathbb{R}$ representing the electric consumption, generation and transmission values. For example, agents 1 and 2 are the supplier and consumer in Region 1, and their generation and consumption values are given by $x_{1}$ and $x_{2}$, respectively. Agent 3 is the distributor which transmits the electricity from Region 1 to 2 , and the transmission value is given by $x_{3}$. The behavior of each agent is described by the utility function $k_{i}\left(x_{i}-x_{i}^{*}\right)^{2}$ with the desired value $x_{i}^{*}$ and price sensitivity $k_{i}>0$. Then, the objective function of the whole system is given by the sum of the agents' utility functions as

$$
F(x)=\sum_{i=1}^{17} k_{i}\left(x_{i}-x_{i}^{*}\right)^{2} .
$$

This grid is divided into some regions, each of which is managed to maintain the supply-demand balance by pricing. Then, the constraint function representing the supply-demand balance is given by

$$
G(x)=\left[\hat{G}(x)^{\top}-\hat{G}(x)^{\top}\right]^{\top}
$$




$$
\hat{G}(x)=\left[\begin{array}{c}
x_{1}-x_{2}-x_{3}+x_{4} \\
x_{3}+x_{5}-x_{6}+x_{7}+x_{8}+x_{9} \\
-x_{7}+x_{10}-x_{11}+x_{12} \\
-x_{8}-x_{12}+x_{13} \\
-x_{4}-x_{9}+x_{14}-x_{15} \\
x_{15}+x_{16}-x_{17}
\end{array}\right]
$$

where each term in $\hat{G}(x)$ represents the surplus or shortage electricity in each region. To keep the supply-demand balance in all the regions, $\hat{G}(x)=0$, namely $G(x) \geq 0$, should be satisfied. The functions $F(x)$ and $G(x)$ in (29) and (30) are obviously convex. Moreover, because $G(x)$ is linear and the constraint condition $G(x) \leq 0$ is satisfied for $x=0$, the strong duality holds [28].

Now, we solve the optimization problem (1) with (29) and (30). Then, $F(x)$ and $G(x)$ are of the forms (21) and (22) with

$$
\begin{aligned}
& \bar{F}^{\{i\}}\left(x_{i}\right)=k_{i}\left(x_{i}-x_{i}^{*}\right)^{2} \\
& \bar{G}^{\{1\}}\left(x_{1}\right)=\left[\begin{array}{llllllllllll}
x_{1} & 0 & 0 & 0 & 0 & 0 & -x_{1} & 0 & 0 & 0 & 0 & 0
\end{array}\right]^{\top} \\
& \bar{G}^{\{2\}}\left(x_{2}\right)=\left[\begin{array}{llllllllllll}
-x_{2} & 0 & 0 & 0 & 0 & 0 & x_{2} & 0 & 0 & 0 & 0 & 0
\end{array}\right]^{\top} \\
& \bar{G}^{\{3\}}\left(x_{3}\right)=\left[\begin{array}{llllllllllll}
-x_{3} & x_{3} & 0 & 0 & 0 & 0 & x_{3} & -x_{3} & 0 & 0 & 0 & 0
\end{array}\right]^{\top} \\
& \bar{F}^{\mathcal{I}}\left(x_{\mathcal{I}}\right)=0, \bar{G}^{\mathcal{I}}\left(x_{\mathcal{I}}\right)=0 \text { if }|\mathcal{I}| \geq 2 .
\end{aligned}
$$

9 Moreover, the graph in Fig. 3 is connected. Thus, the conditions in Theorem 1 are fulfilled, and the distributed constraint optimization problem is solvable by using the proposed method. Note that (23) is corresponding to the agent behavior model, and that (26) and (27) present the pricing law of the electricity because the Lagrangian multiplier represents the price. Then, this update law gives a rule to determine an appropriate price via negotiations between agents. The negotiations are carried out through the information exchanged over the graph given by Fig. 3. This pricing system cannot be established by the conventional distributed optimization methods, e. g. $[13,14]$, which require supervisors to determine the Lagrangian multiplier.

The simulation results are depicted in Figs. 4, 5 and 6, which show the values of $x_{i}^{[s]}, F\left(x^{[s]}\right)$ and $G\left(x^{[s]}\right)$ for step $s$. It is observed that $F\left(x^{[s]}\right)$ is monotonically decreasing while $G\left(x^{[s]}\right)$ converges to 0 . Fig. 7 shows the Lagrangian multiplier $\lambda^{[s]}$, which is the result of the individual computation of 
each agent according to (26) and (27). Fig. 8 shows the transition of the computation, where the first components of $\hat{\lambda}_{i}[k]$ for $l \in \mathbb{Z}_{+}$at a certain step $s$ are depicted. The doted line represents the appropriate value of $\lambda^{[s+1]}$ given by (11). This figure shows that the agents successfully estimate the Lagrangian multiplier in a distributed manner. As a result, the supply-demand balance is maintained by the distributed pricing. This result illustrates the effectiveness of the proposed method.

\section{Conclusion}

In this paper, we considered the distributed constraint optimization problem on networked multi-agent systems. We proposed a distributed method to estimate the Lagrangian multiplier, which is executable on each agent by using only local information over the network. Then, a necessary and sufficient condition was derived for the distributed constraint optimization problem to be solvable. Finally, we applied this method to power grid control to maintain the supply-demand balance by pricing. The proposed distributed update law of the Lagrangian multiplier gives a pricing rule for the agents to determine an appropriate price by negotiations. As for Remark 1, the number of the iteration in the proposed algorithm should be determined according to the tolerance optimization error. To determine an appropriate number is important future work.

\section{Acknowledgment}

This research was partly supported by CREST/JST.

\section{References}

[1] F. P. Kelly, A. K. Maulloo, D. K. H. Tan, Rate control for communication networks: Shadow prices, proportional fairness and stability, Jour. of the Operational Research Society 49 (3) (1998) 237-252.

[2] S. H. Low, D. E. Lapsle, Optimization flow control-I: Basic algorithm and convergence, IEEE/ACM Trans. on networking 7 (6) (1999) 861874 .

[3] S. H. Low, F. Paganini, J. C. Doyle, Internet congestion control, IEEE Control Systems Magazine 22 (1) (2002) 28-43. 
[4] R. Srikant, The Mathematics of Internet Congestion Control, SpringerVerlag, 2004.

[5] E. Campos-Nañez, A. Garcia, C. Li, A game theoretic approach to efficient power management in sensor networks, Operation Research 56 (3) (2008) 552-561.

[6] P. Samadi, A. H. Mohsenian-Rad, R. Schober, V. W. S. Wong, J. Jatskevich, Optimal real-time pricing algorithm based on utility maximization for smart grid, in: First IEEE Int. Conf on Smart Grid Communications, 2010 .

[7] N. Li, L. Chen, S. H. Low, Optimal demand response based on utility maximization in power networks, in: IEEE Power and Energy Society General Meeting, 2011.

[8] L. Gkatzikis, I. Koutsopoulos, T. Salonidis, The role of aggregators in smart grid demand response markets, Selected Areas in Communications 31 (7) (2013) 1247-1257.

[9] B. N. Janson, Dynamic traffic assignment for urban road networks, Transportation Research Part B: Methodological 25 (2-3) (1991) 143161.

[10] S.-W. Chiou, Optimization of area traffic control for equilibrium network flows, Transportation Science 33 (3) (1999) 279-289.

[11] M. Papageorgiou, C. Diakaki, V. Dinopoulou, A. Kotsialos, Y. Wang, Review of road traffic control strategies, Proc. of the IEEE 91 (12) (2003) 2043-2067.

[12] B. Yang, M. Johansson, Distributed Optimization and Games: A Tutorial Overview, Springer-Verlag, 2010.

[13] M. Chiang, S. H. Low, A. R. Calderbank, J. C. Doyle, Layering as optimization decomposition: A mathematical theory of network architectures, Proc. of the IEEE 95 (1) (2007) 255-312.

[14] D. P. Palomar, M. Chiang, A tutorial on decomposition methods for network utility maximization, IEEE Jour. on Selected Areas in Communications 24 (8) (2006) 1439-1451. 
[15] Y. Wakasa, M. Arakawa, K. Tanaka, T. Akashi, Decentralized model predictive control via dual decomposition, in: Proc. of the 47th IEEE Conf. on Decision and Control, 2008.

[16] H. Terelius, U. Topcu, R. M. Murray, Decentralized multi-agent optimization via dual decomposition, in: IFAC World Congress, 2011.

[17] S. Boyd, N. Parikh, E. Chu, B. Peleato, J. Eckstein, Distributed optimization and statistical learning via the alternating direction method of multipliers, Foundations and Trends in Machine Learning 3 (1) (2010) $1-122$.

[18] D. D. N. Chatzipanagiotis, M. M. Zavlanos, An augmented lagrangian method for distributed optimization, Mathematical Programming 152 (1-2) (2015) 405-434.

[19] A. Nedić, A. Ozdaglar, Distributed subgradient methods for multi-agent optimization, Trans. on Automatic Control 54 (1) (2009) 48-61.

[20] A. Nedić, A. Ozdaglar, P. A. Parrilo, Constrained consensus and optimization in multi-agent networks, IEEE Trans. on Automatic Control 55 (4) (2010) 922-938.

[21] I. Lobel, A. Ozdaglar, D. Feijer, Distributed multi-agent optimization with state-dependent communication, Mathematical Programming, Ser. B 129 (2) (2011) 255-284.

[22] M. Zhu, S. Martínez, An approximate dual subgradient algorithm for multi-agent non-convex optimization, IEEE Trans. on Automatic Control 58 (6) (2013) 1534-1539.

[23] T.-H. Chang, A. Nedić, A. Scaglione, Distributed constrained optimization by consensus-based primal-dual perturbation method, IEEE Trans. on Automatic Control 59 (6) (2014) 1524-1538.

[24] D. G. Luenberger, Linear and Nonlinear Programming, 2nd Edition, Kluwer Academic Publishers, 2003.

[25] A. Jadbabaie, A. Ozdaglar, M. Zargham, A distributed newton method for network optimization, in: Proc. of the 48th IEEE Conf. on Decision and Control, 2009. 
1 [26] K. Sakurama, S. Azuma, T. Sugie, Distributed controllers for multiagent coordination via gradient-flow approach, IEEE Trans. on Automatic Control 60 (6) (2015) 1471-1485.

4 [27] R. Olfati-Saber, R. M. Murray, Consensus problems in networks of agents with switching topology and time-delays, IEEE Trans. on Automatic Control 49 (9) (2004) 1520-1533.

7 [28] S. Boyd, L. Vandenberghe, Convex Optimization, Cambridge University Press, 2004. 


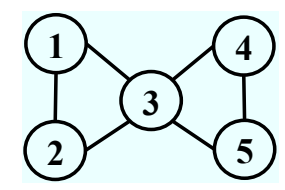

Figure 1: Example of a graph 


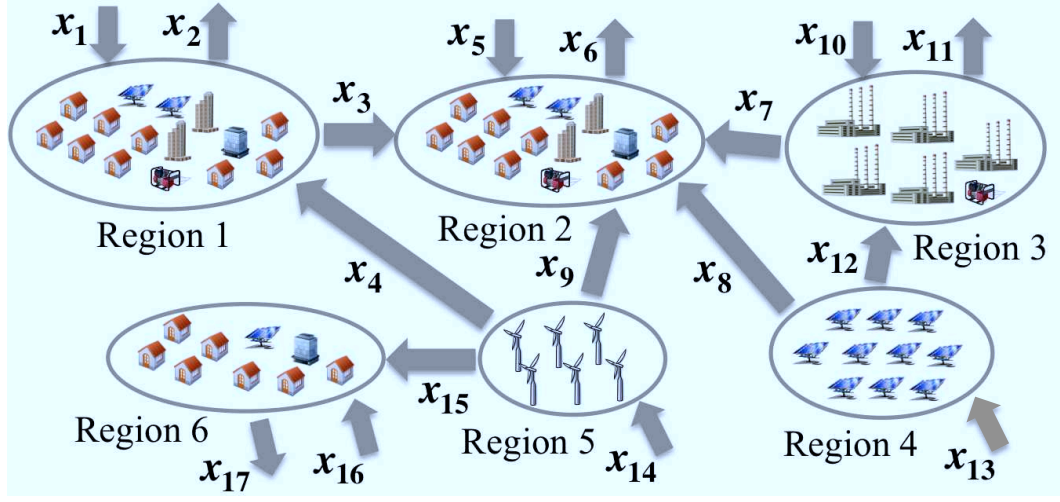

Figure 2: Power grid 


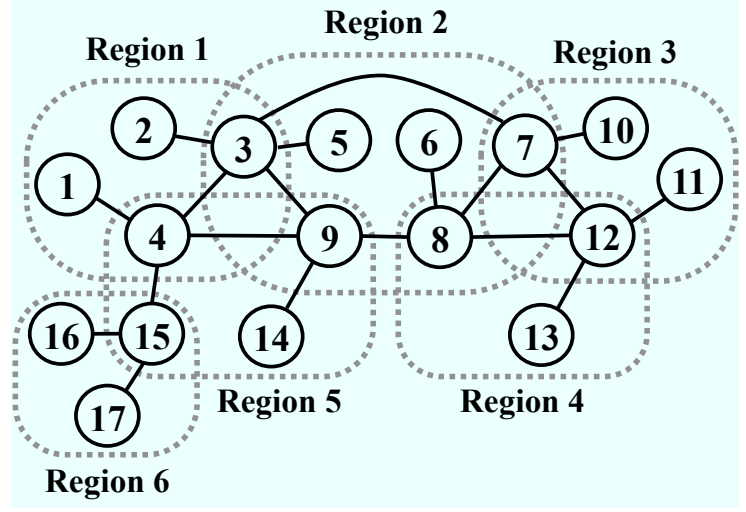

Figure 3: Communication graph for pricing 


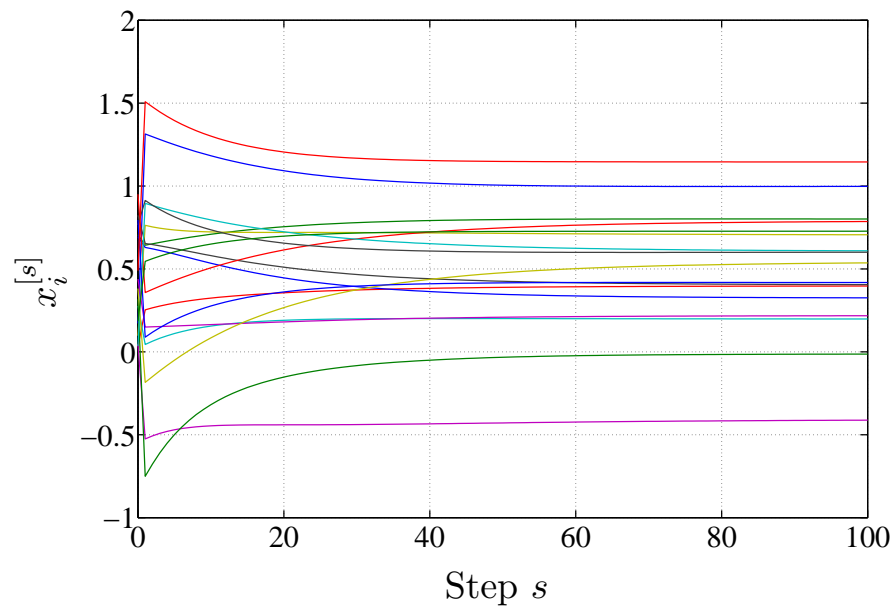

Figure 4: Variable $x_{i}^{[s]}$ of each agent $i \in \mathcal{V}$ 


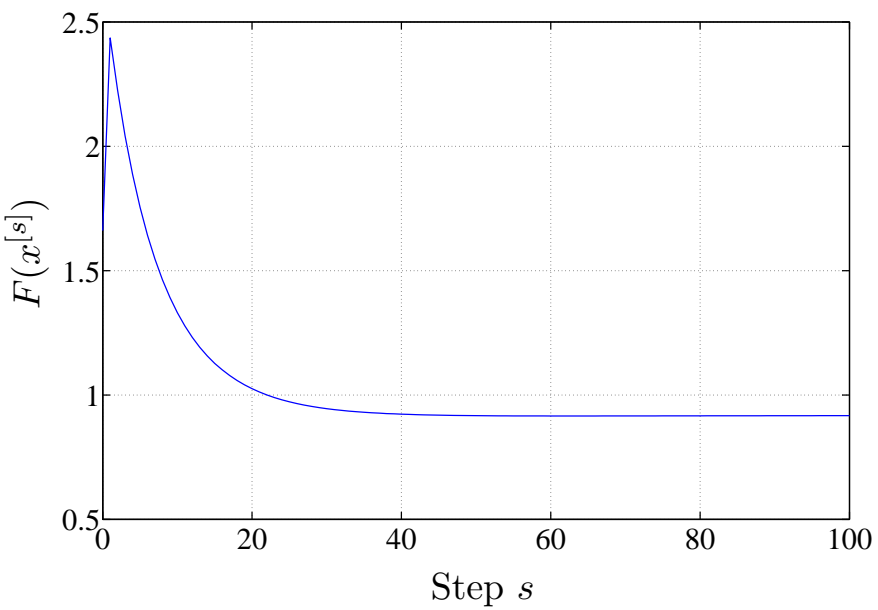

Figure 5: Objective function $F\left(x^{[s]}\right)$ 


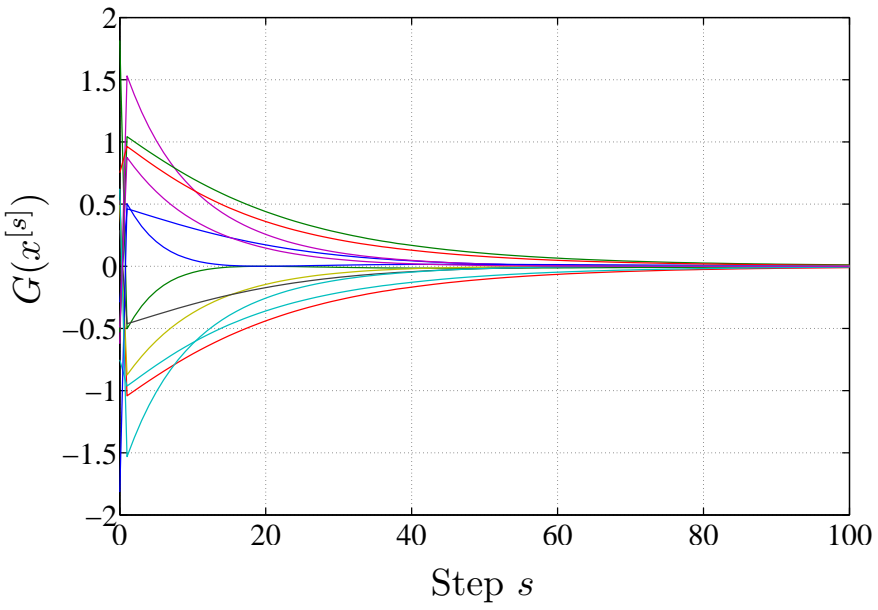

Figure 6: All components of constraint function $G\left(x^{[s]}\right)$ 


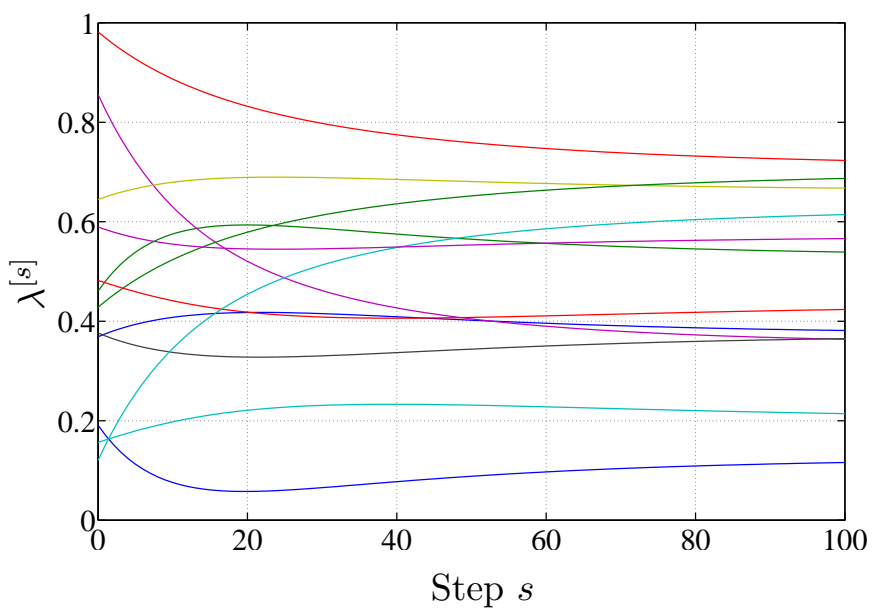

Figure 7: All components of Lagrange multiplier $\lambda^{[s]}$ 


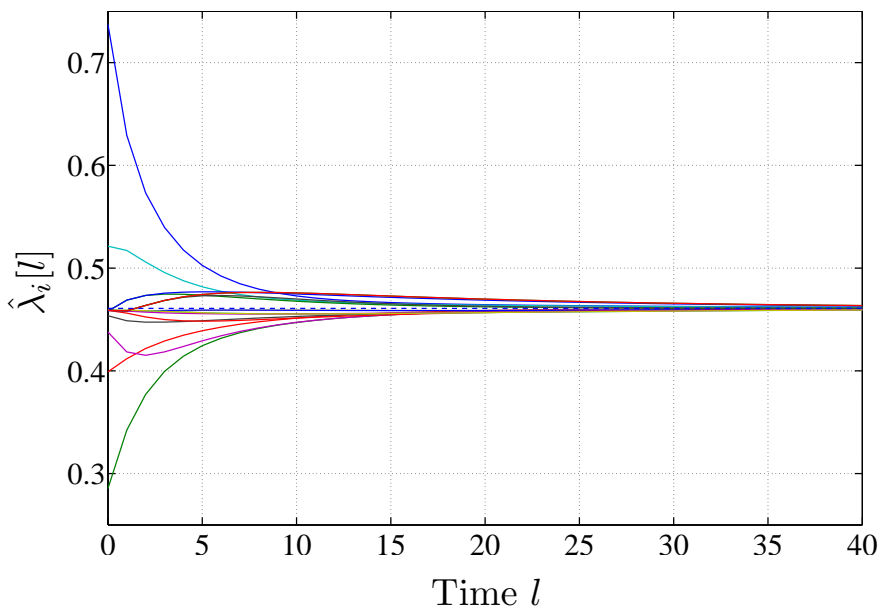

Figure 8: First component of $\hat{\lambda}_{i}[l]$ for each $i \in \mathcal{V}$ 